\title{
Chest imaging by high-resolution computed tomography (HRCT): Diagnosis
}

S K Misser, MB ChB, FCRad (D) SA

Lake Smit and Partners, Durban

\section{A Abdullah, MB ChB, FCP (SA), FCCP}

St Augustine's Hospital, Durban

Corresponding author: S Misser (misser@lakesmit.co.za)

We congratulate Dr Qonita Said-Hartley, consultant radiologist at New Somerset Hospital, Cape Town, for a most detailed diagnosis for which she receives an award of R1 000 from the RSSA. The two runners-up - Dr Kamila Padia and Dr Marc Jordaan - warrant honourable mention. Dr Misser elaborates below on the condition and its imaging. Please refer to page 79 of the June 2012 issue of the SAJR (http://www.sajr.org.za/index.php/sajr/article/view/729/566) for the presentation details (a 39-year-old man with subacute dyspnoea and intermittent cough) and images. The previously published and two new images appear below

\section{Diagnosis}

The plain radiograph demonstrates multi-segment bilateral lung opacities with associated fine nodular infiltration. The chemotherapy port in situ relates to prior treatment for colonic cancer. Cardiomediastinal silhouette and pulmonary vascularity are normal. Serial level HRCT images reveal diffuse centrilobular nodular infiltrates and ground glass opacification (GGO) in both lung fields.
Patchy areas of involvement with adjacent normal-appearing lung segments are noted. This is in keeping with a mosaic pattern of attenuation. No air trapping was detected on expiration. Pulmonary vessels are of uniform calibre throughout the lung. The areas of GGO are therefore the abnormal finding, and the low attenuation areas are normal intervening lung segments. In the absence of marked superimposed reticulation, the pathology suspected is parenchymal lung disease rather than predominant interstitial or lymphatic process.

Further questioning revealed that the patient was a pigeon breeder with more than 100 birds in his aviary. The chemotherapy port was a red herring. The possibility of post-chemotherapy- (infective or immunologic) related lung disease was thought a less likely differential diagnosis. A diagnosis of subacute hypersensitivity (Bird fancier's) pneumonitis was made. He was treated with a short course of steroids and advised to reduce exposure to birds. Follow-up study showed complete resolution of the allergic alveolitis changes - see Figs 4 and 5 above, demonstrating before- and after-treatment images.

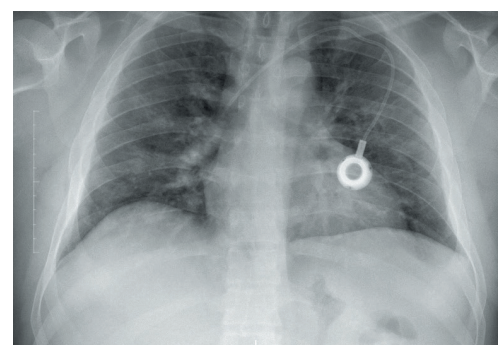

Fig. 1. Plain PA chest radiograph.

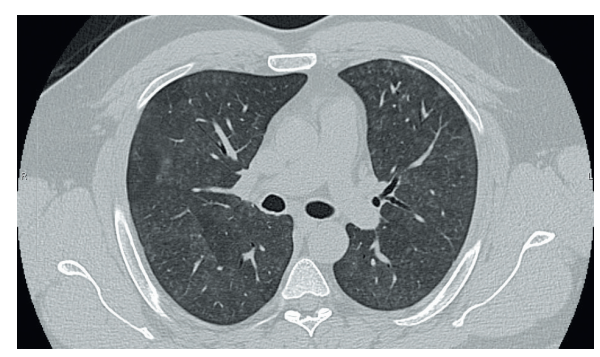

Fig. 2. Axial HRCT at pulmonary artery level.

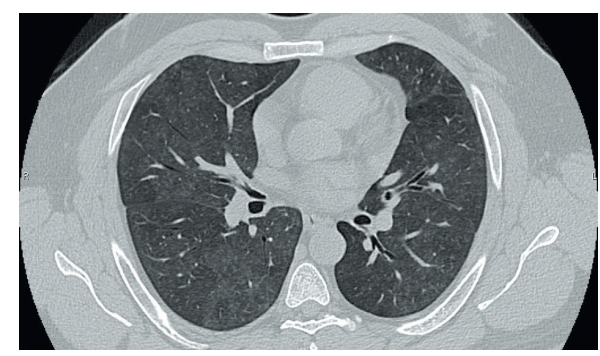

Fig. 3. Axial HRCT at aortic root level.
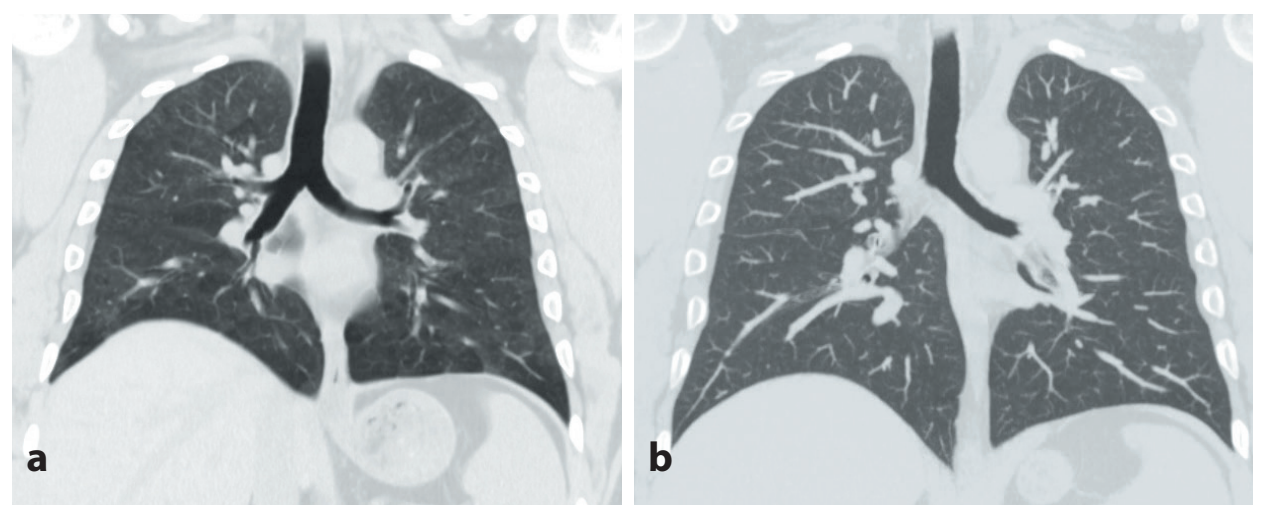

Figs $4 a$ and $4 b$. Coronal reformatted CT images before and after steroid therapy. 

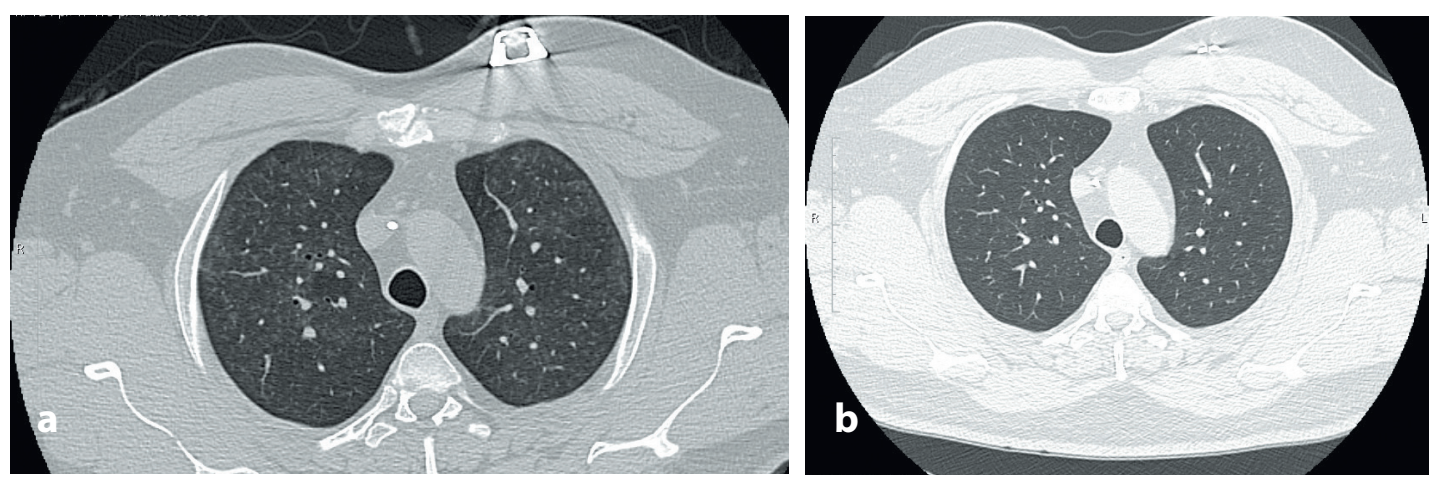

Figs $5 a$ and $5 b$. Axial CT images before and after steroid therapy.

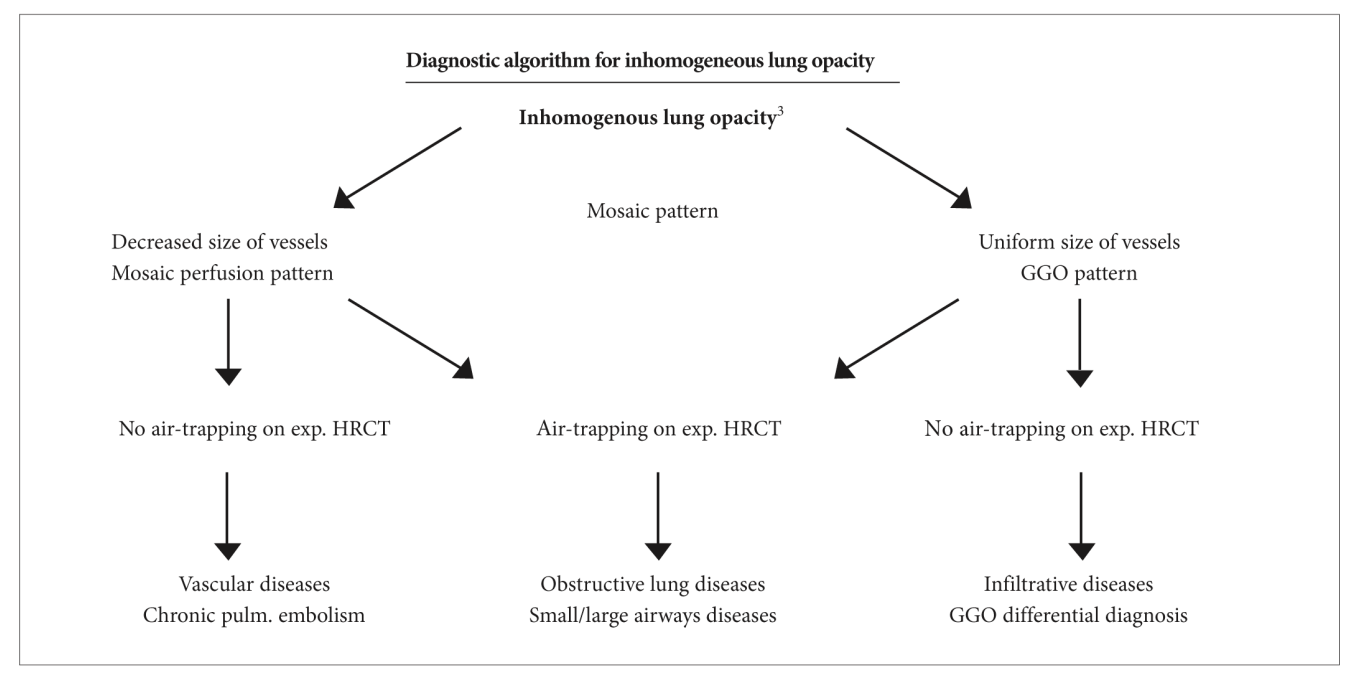

Hypersensitivity pneumonitis (HP), originally known as extrinsic allergic alveolitis, is an allergic lung disease caused by the inhalation of organic or inorganic particles that trigger a cascade of immune-complex and cell-mediated reactions. Bird fancier's lung is one of the most common forms of HP. ${ }^{1}$ It is a unique form of HP in that the offending antigen is a protein, rather than a micro-organism, and is contained in the feathers, serum and guano of birds. ${ }^{2}$ Imaging usually undertaken in the subacute phase of the disease reveals ill-defined small centrilobular

\section{Table 1. Subacute HP HRCT findings ${ }^{1,3}$}

- Small centrilobular nodular opacities

- Isolated or diffuse GGO

- Superimposition of first two findings

- Lobular areas of decreased/mosaic attenuation

- Lobular areas of air-trapping

\section{Table 2. Peripheral/patchy GGO ${ }^{3}$}

- Hypersensitivity pneumonitis

- Interstitial pneumonia (UIP, NSIP, DIP)

- Alveolar proteinosis

- Eosinophilic pneumonia

- Sarcoidosis

- Organising pneumonia nodules and a mosaic pattern (Table 1). Treatment is usually supportive and antigen avoidance in the acute/subacute phase is generally all that is needed.

\section{Approach to mosaic pattern of attenuation}

Ground-glass opacity (GGO) is defined as a hazy increase in lung density with preservation of airway and vessel margins. A mosaic pattern refers to the presence of patchy areas of GGO interspersed with adjacent darker areas. The radiologist has to determine if the area of increased lung opacity is abnormal or if the darker areas are abnormal. A key step is the assessment of the calibre of the pulmonary vessels in the areas of inhomogeneous lung opacity. (See diagnostic algorithm below.)

When the pulmonary vessels are uniform in size, this is probably due to parenchymal lung disease (Table 2). Decreased size of the pulmonary vessels is cause to suspect mosaic perfusion. The pattern may be due to vascular causes (e.g. chronic pulmonary thrombo-embolism) or airway obstruction with redistribution of blood to the normal hyper-attenuated areas (e.g. bronchiolitis obliterans). An expiratory CT scan assists in dividing these patterns further. Those with air trapping demonstrate further relative darkening of the low attenuation areas.

1. Silva CI, Churg A, Müller NL. Hypersensitivity pneumonitis: Spectrum of high-resolution CT and pathologic findings. AJR 2007;188(2):224-244.

2. Chan AL, Juarez MM, Leslie KO, Ismail HA, Albertson TE. Bird fancier's lung: A state of the art review. Clin Rev Allergy Immunol 2012;43(1-2):69-83.

3. Webb WR, Muller NL, Naidich DP. High-Resolution CT of the Lung. 4th ed. Philadelphia, USA: Lippincott Williams \& Wilkins, 2008. 